\title{
Delivering the goods: realizing the clinical potential of RNAi
}

"The clinical feasibility of RNAi therapeutics will depend on the effectiveness, safety and cost. Nanoparticles capable of site-specific and intracellular delivery combined with optimal RNA design are needed to maximize the therapeutic efficacy to reduce dosage and nonspecific effects."

The ability to utilize synthetic siRNA duplexes to interrupt gene expression has propelled RNAi to the forefront of biomedical research both as a tool to study cellular processes and as a genetic medicine to silence genes implicated in human disease [1]. Greater understanding of the complex molecular mechanisms of RNAi has made an expanding repertoire of potential RNAi-based drugs available that includes conventional, Dicer-substrate siRNA (DsiRNA) [2] and transcriptional silencing siRNA (tsRNA) [3]. Furthermore, an intense effort is now centered on the identification of therapeutic miRNA candidates, capable of regulating expression of multiple target genes by inducing mRNA cleavage and/or translational inhibition [4]. The target-specific nature of action offers reduced toxicity but the clinical worth of these, as with other bioactive molecules, is determined by the ability to disseminate in the body and reach target sites at therapeutically relevant levels. The poor pharmacokinetic profile, as a consequence of the susceptibility of siRNA for serum degradation, renal clearance and nonspecific accumulation, restricts systemic applications. In RNAi clinical trials taken through into Phase II, siRNA administration directly into ocular and nasal sites circumvents any necessity for systemic administration. Local application, however, limits the treatment to easily accessible diseases. The fact remains that delivery is a key determinant in realizing the full clinical potential of RNAi. In the context of RNAi, delivery refers also to intracellular trafficking to allow interaction with the RNAi machinery (e.g., RISC, DICER or Drosha) and the mRNA target. Therefore, RNAi-based therapies are dependent on the development of technologies able to overcome both extracellular and intracellular delivery requirements. This need is highlighted by the intense pursuit and investment undertaken by
Big Pharma for delivery solutions to pave the way to capitalize on the enormous potential of RNAi technology.

The predisposition for nanometric particles to circumvent the renal clearance mechanism and yet permit size-mediated transfer across vasculature endothelium into the tissue promotes their use for systemic delivery of siRNA. Cationic lipid- and polymer-based nanoparticles have been developed to improve the therapeutic potential of RNAi-based agents, providing protection from serum degradation and facilitating site-specific delivery, cellular uptake and intracellular trafficking. Polycation-based nanoparticles (also termed polyplexes) formed by self-assembly of siRNA with synthetic or natural polycations are promising RNAi-based nanomedicines [5]. Flexibility in design and versatility is their hallmark; with a capability to introduce different functional groups into a wide range of polymer types, multifunctional properties needed to fulfill delivery requirements can be added. Surface modification with hydrophilic polymers such as poly(ethylene glycol) (PEG) during polyplex self-assembly has been used for steric stabilization and results in 'stealth-like' nanoparticles that reduce serum protein interactions and capture by the mononuclear phagocyte system. Tumor-specific gene silencing has been demonstrated after intravenous injection of PEGylated polyethylenimine (PEI) and cyclodextrin nanoparticles re-targeted with RGD peptide [6], transferrin [7] and VEGF [8] ligands. SiRNA/antibodyprotamine fusion protein complexes exhibit tumor-targeted systemic silencing in the absence of PEGylation, thus challenging the dogma that PEGylation and targeting is a necessary combination. Peer and coworkers have developed a targeted stabilized nanoparticle system composed of protamine/siRNA incorporated within a neutral phospholipid-based

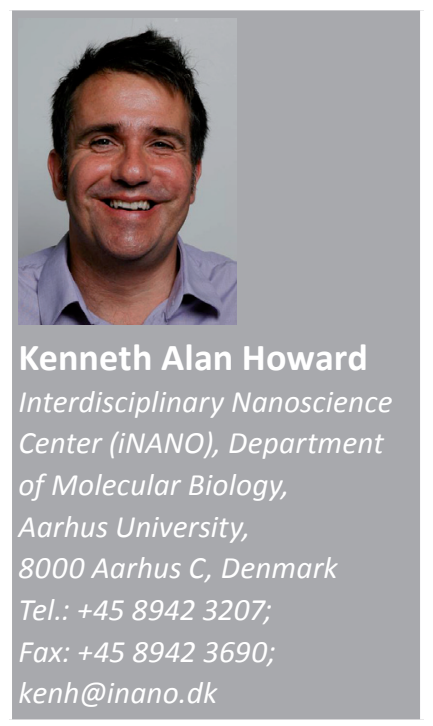

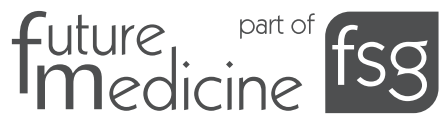


unilamellar vesicle coated with a hyaluronanantibody conjugate [9]. Cellular targeting and internalization by antibody recognition of the $\beta 7$ integrin on gut mononuclear leukocytes resulted in reduced siRNA-specific cyclin D1 expression and reversed colitis in mice. The in vivo stabilization effect probably reflects a reduction in serum protein interactions due to the anionic hyaluronan surface.

“...an intense effort is now centered on the identification of therapeutic miRNA candidates, capable of regulating expression of multiple target genes by inducing $m R N A$ cleavage and/or trans/ational inhibition."

In the majority of preclinical studies, systemic silencing results from passive accumulation and physiological effects rather than design optimized for targeting. The systemic gene silencing shown in preclinical models for cancer [10] and inflammation [11] that occurs without PEGylation or active targeting is probably due to the enhanced permeability and retention effect by which nanoscale particles migrate and accumulate across leaky vasculature in disease tissue. Liver deposition of nanoparticles, associated with a discontinuous endothelial barrier and phagocytic capture by fixed tissue macrophages, may contribute to the ability of polymers [12] and lipid-based nanoparticles [13] to mediate reduction in cholesterol blood levels through silencing of ApoB. Moreover, proteinmediated aggregation of cationic nanoparticles and subsequent physical entrapment within pulmonary capillary beds could account for effective pulmonary silencing for systemic $\mathrm{PEI} / \mathrm{siRNA}$ polyplexes [14]. Dependence on physiological conditions and passive targeting limits the capacity to control and limits the potential of delivery. The widespread biodistribution and nonspecific nature commonly associated with nonmodified nanoparticles can increase the dose requirement and potential side effects. Reduced mononuclear phagocyte system-mediated clearance, which occured in immunocompromised mice used in a number of studies, enables improved nanoparticle delivery, however, this is not applicable in the clinical setting. There is a call for more advanced stealth and targeting technology to control site-specific homing, which is still an unresolved issue in nanoparticle-based drug delivery. Issues such as precise design of surface structure, density of hydrophilic polymers and optimal presentation of target moieties need to be addressed.
For certain disease types it makes sense to use local delivery. RNAi-based treatments based on local mucosal application would be more effective than the systemic route for conditions associated with the mucosal surfaces lining the respiratory, gastrointestinal and genitourinary tracts. Delivery by this route offers more likelihood of clinical success in the short term, with intense attention focused on pulmonary gene silencing with naked, lipid and polycation-based systems. Bioadhesive polymers such as chitosan can be used to overcome the physical barrier and mucociliary clearance of tightly packed ciliated epithelial cells and overlying mucus for delivery of drugs. The mucoadehesive and mucoperameable properties of chitosan have been used for pulmonary gene silencing using a chitosan/siRNA nanoparticle system [15].

Endosomolytic and cleavable polymers can be built into the design of siRNA polyplexes to facilitate cytosolic release of siRNA needed to permit siRNA interaction with the intracellular target. Endosomal buffering, $\mathrm{pH}$-activated polymers and membrane interactive peptides, which allow endosomal escape and transport to the cytosol, can be used to overcome capture within the endosomal-lysosomal pathway associated with cellular endocytosis of nanoparticles. The advent of bioresponsive nanoparticles whose function is triggered by biological conditions is a method used to control spatial delivery. Nanoparticles composed of reducible disulfidelinked polycations cleaved in response to intracellular redox conditions is an exciting strategy to install extracellular stability whilst allowing for intracellular breakdown and maximal release of the siRNA cargo.

\section{"RNAi-based therapies are dependent on the development of technologies able to overcome both extracellular and intracellular delivery requirements."}

New classes of RNAi-based drugs including primary miRNA transcripts (pri-miRNA) and tsRNA require nuclear localization for interaction with RNAi machinery or targets, compared with cytoplasmic localization for conventional and DsiRNA. A polyplex composed of a reducible copolypeptide of a histidine-rich peptide and a nuclear localization signal (NLS) peptide has been used for pri-miRNA and tsRNA delivery [16]. Histidyl residues promote endosomal escape by the proton sponge mechanism due to the buffering capacity of histidyl at endosomal $\mathrm{pH}$ whilst disulfide bridges allow intracellular dissembly. 
This is an example of a multicomponent system displaying the combined functions of endosomal escape, nuclear localization and nanoparticle dissembly. NLS peptide-dependent pri-miRNA processing and tsRNA-mediated silencing in vitro was demonstrated with this system. The level of nuclear localization determined by confocal microscopy was optimized using specific histidine:NLS copolypeptide ratios showing the necessity for correct histidine/NLS peptide balance for nanoparticle stability and nuclear trafficking. The added necessity for delivery across the nuclear membrane is outweighed by the reduced nonspecific induction of innate immunity and prolonged silencing activity associated with miRNA and tsRNA, respectively.

\section{"The limited success of antisense and plasmid-based genetic medicine is partly a consequence of delivery failures; however, the avalanche of research, investment and expectations in RNAi might just drive through the delivery advances needed to realize the clinical potential of RNAi medicines."}

The duration of gene silencing and nonspecific innate immune effects are important parameters in RNAi-based therapies. Synthetic RNA duplexes are able to elicit silencing over a few days, whilst plasmid-driven intracellular expression of shRNA can last a number of weeks. The advantage of synthetic RNA duplexes from a clinical perspective is accurate control of the administered dose and the ability for chemical modification to improve stability and reduce nonspecific inflammatory effects. Recent evidence suggests nonspecific induction of innate inflammatory responses, such as type I IFN resulting from siRNA interaction with endosomal Tolllike receptors (TLRs), may be responsible for the therapeutic effects observed in disease animal models supported by the nonimmunostimulatory-enhanced green fluorescent protein sequence used routinely as a negative control $[17,18]$. This has worrying implications for nanoparticlebased RNAi treatments considering the fact that nanoparticles potentiate delivery into endosomal compartments. Therefore, it is important to consider siRNA modification in combination with nanoparticle formulation to limit induction of innate immunity. Endosomolytic polymers that allow endosomal escape or incorporation of modified nucleotides that inhibit TLR interaction can be used. The relationship between and influence of siRNA design and nanoparticle formulation on nonspecific effects has been addressed in our work [19]. Lower levels of type I IFN production in harvested peritoneal macrophages from mice has been achieved after intraperitoneal injection of chitosan nanoparticles containing 2'-O-methyl modified anti-TNF- $\alpha$ DsiRNA compared with an unmodified duplex, likely by blocking TLR interaction by chemical modification [20]. Taken forward into a murine collagen-induced arthritis model, the lowest arthritic scores were obtained with modified DsiRNA-containing nanoparticles that correlate with reduced type I IFN. Equal consideration to both siRNA design and nanoparticle formulation is a vital requirement for RNAi-based therapies.

Polymers (and also lipids) can influence cellular gene expression [21], which may interfere with the specificity of treatment, so it is important to screen for possible cellular effects for each type of nanoparticle system using microarray analysis. The issues of safety and standardized production are important for clinical development. The manufacture of synthetic polymers produces polymers with the defined molecular weight and low polydispersity required for the clinic. The application of reducible polycations that breakdown inside the cell into low-molecular-weight fractions is a potential strategy to circumvent the toxicity associated with some high-molecular-weight synthetic polymers. The cyclodextrin-based siRNA system RONDEL ${ }^{\mathrm{TM}}$ produced by Calando Pharmaceuticals is the first polycation nanoparticle set for RNAi Phase I clinical trials as an intravenous treatment for solid tumors. The system is based on polycations of natural cyclodextrin that contain a lypophilic central cavity used to anchor adamantine-PEG or adamantane-PEG-transferrin conjugates after self-assembly with siRNA into nanoparticles. Impressive transferrin-targeted delivery and antitumor effects in preclinical models for cancer [22] and high tolerance in nonhuman primates [23] support the clinical application for this system.

\section{"Equal consideration to both siRNA design and nanoparticle formulation is a vital requirement for RNAi-based therapies."}

The clinical feasibility of RNAi therapeutics will depend on the effectiveness, safety and cost. Nanoparticles capable of site-specific and intracellular delivery combined with optimal RNA design are needed to maximize the therapeutic efficacy to reduce dosage and nonspecific effects. Multidisciplinary research at the molecular biology/pharmaceutical/nanotechnology interface is needed to develop these new nanomedicines. 
This editorial has focused on polycation-based nanoparticle systems but the delivery and technology requirements also apply to alternatives such as lipid-based methods. The limited success of antisense and plasmid-based genetic medicine is partly a consequence of delivery failures; however, the avalanche of research, investment and expectations in RNAi might just drive through the delivery advances needed to realize the clinical potential of RNAi medicines.
Financial \& competing interests disclosure

The author has no relevant affliations or financial involvement with any organization or entity with a financial interest in or financial conflict with the subject matter or materials discussed in the manuscript. This includes employment, consultancies, honoraria, stock ownership or options, expert testimony, grants or patents received or pending, or royalties.

No writing assistance was utilized in the production of this manuscript.

\section{Bibliography}

1 de Fougerolles A, Vornlocher HP, Maraganore J, Lieberman J: Interfering with disease: a progress report on siRNA-based therapeutics. Nat. Rev. Drug. Discov. 6, 443-453 (2007).

2 Kim DH, Behlke MA, Rose SD, Chang MS, Choi S, Rossi JJ: Synthetic dsRNA Dicer substrates enhance RNAi potency and efficacy. Nat. Biotechnol. 23, 222-226 (2005).

3 Morris KV, Chan SW, Jacobsen SE, Looney DJ: Small interfering RNA-induced transcriptional gene silencing in human cells. Science 305, 1289-1292 (2004).

4 He L, Hannon GJ: MicroRNAs: small RNAs with a big role in gene regulation. Nat. Rev. Genet. 5, 522-531 (2004).

5 Howard KA: Delivery of RNA interference therapeutics using polycation-based nanoparticles. Adv. Drug Deliv. Rev. 61, 710-720 (2009).

6 Schiffelers RM, Ansari A, Xu J et al.: Cancer siRNA therapy by tumor selective delivery with ligand-targeted sterically stabilized nanoparticle. Nucleic Acids Res. 32, e149 (2004).

7 Bartlett DW, Davis ME: Impact of tumor-specific targeting and dosing schedule on tumor growth inhibition after intravenous administration of siRNA-containing nanoparticles. Biotechnol. Bioeng. 99, 975-985 (2008).

8 Kim SH, Jeong JH, Lee SH, Kim SW, Park TG: Local and systemic delivery of VEGF siRNA using polyelectrolyte complex micelles for effective treatment of cancer. J. Control. Release 129, 107-116 (2008).
9 Peer D, Park EJ, Morishita Y, Carman CV, Shimaoka M: Systemic leukocyte-directed siRNA delivery revealing cyclin D1 as an anti-inflammatory target. Science 319, 627-630 (2008).

10 Takeshita F, Minakuchi Y, Nagahara S et al: : Efficient delivery of small interfering RNA to bone-metastatic tumors by using atelocollagen in vivo. Proc. Natl Acad. Sci. USA 102, 12177-12182 (2005).

11 Ishimoto T, Takei Y, Yuzawa Y et al.: Downregulation of monocyte chemoattractant protein-1 involving short interfering RNA attenuates hapten-induced contact hypersensitivity. Mol. Ther. 16, 387-395 (2008).

12 Baigude H, Mccarroll J, Yang CS, Swain PM, Rana TM: Design and creation of new nanomaterials for therapeutic RNAi. ACS Chem. Biol. 2, 237-241 (2007).

13 Zimmermann TS, Lee AC, Akinc A et al.: RNAi-mediated gene silencing in non-human primates. Nature 441, 111-114 (2006).

14 Thomas M, Lu JJ, Ge Q, Zhang C, Chen J, Klibanov AM: Full deacylation of polyethylenimine dramatically boosts its gene delivery efficiency and specificity to mouse lung. Proc. Natl Acad. Sci. USA 102, 5679-5684 (2005).

15 Howard KA, Rahbek UL, Liu X et al.: RNA interference in vitro and in vivo using a novel chitosan/siRNA nanoparticle system. Mol. Ther. 14, 476-484 (2006).

16 Rahbek UL, Howard KA, Oupicky D et al:: Intracellular siRNA and precursor miRNA trafficking using bioresponsive copolypeptides. J. Gene Med. 10, 81-93 (2008).
17 Robbins M, Judge A, Ambegia E et al.: Misinterpreting the therapeutic effects of small interfering RNA caused by immune stimulation. Hum. Gene Ther. 19, 991-999 (2008).

18 Robbins M, Judge A, Maclachlan I: siRNA and innate immunity. Oligonucleotides 19 , 89-102 (2009).

19 Howard KA, Paludan SR, Behlke MA, Besenbacher F, Deleuran B, Kjems J: Chitosan/siRNA nanoparticle-mediated TNF- $\alpha$ knockdown in peritoneal macrophages for anti-inflammatory treatment in a murine arthritis model. Mol. Ther. 17, 162-168 (2009).

20 Judge AD, Bola G, Lee AC, Maclachlan I: Design of noninflammatory synthetic siRNA mediating potent gene silencing in vivo. Mol. Ther. 13, 494-505 (2006).

21 Akhtar S, Benter I: Toxicogenomics of non-viral drug delivery systems for RNAi: potential impact on siRNA-mediated gene silencing activity and specificity. Adv. Drug Deliv. Rev. 59, 164-182 (2007).

22 Hu-Lieskovan S, Heidel JD, Bartlett DW, Davis ME, Triche TJ: Sequence-specific knockdown of EWS-FLI1 by targeted, nonviral delivery of small interfering RNA inhibits tumor growth in a murine model of metastatic Ewing's sarcoma. Cancer Res. 65 , 8984-8992 (2005).

23 Heidel JD, Yu Z, Liu JY et al.: Administration in non-human primates of escalating intravenous doses of targeted nanoparticles containing ribonucleotide reductase subunit M2 siRNA. Proc. Natl Acad. Sci. USA 104, 5715-5721 (2007). 